\title{
Clinical decision making: an introduction
}

\author{
Giovanni Casazza · Giorgio Costantino • \\ Piergiorgio Duca
}

Received: 20 September 2010/Accepted: 20 October 2010/Published online: 13 November 2010

(C) SIMI 2010

\section{Introduction}

An 80-year-old man, affected by chronic obstructive pulmonary disease (COPD), came to our attention complaining of shortness of breath and pleuritic right posterior chest pain lasting $3 \mathrm{~h}$. In the prior week, he was almost bedridden. The past history included a mild cognitive impairment and obesity. His relatives reported cough without fever, there was no trauma. The clinical examination revealed mild pitting oedema of the legs, decreased breath sounds, heart rate 102 beats/min., oxygen saturation $92 \%$ and blood pressure 135/80. A chest X-ray study suggested pneumonia in the left lung.

The physician in charge of such a patient faces many possibilities: which diagnosis is the right one between pneumonia, COPD with acute exacerbation, heart failure and pulmonary embolism (PE). What diagnostic tests should be ordered first to exclude or confirm the diagnosis, and finally what therapy should be undertaken?

The clinician has to decide whether to carry on further clinical investigations or to initiate a treatment without knowing the true diagnosis. One must take into account the probability of the disease, its severity, and, finally, the balance between benefits and harms of the treatment. In

G. Casazza ( $\bowtie)$

Sede di Via Venezian del Dipartimento di Medicina del Lavoro, Università degli Studi di Milano,

Via Venezian 1, 20133 Milan, Italy

e-mail: giovanni.casazza@unimi.it

G. Costantino

Medicina Interna II, Ospedale "L. Sacco" Milano, Milan, Italy

P. Duca

Dipartimento di Scienze Cliniche,

Università degli Studi di Milano, Milan, Italy some cases, the decisions are intuitive, when for serious diseases, for example, an effective treatment exists without any relevant side effects. As an example, Folic acid supplements during pregnancy dramatically reduce any risk of spina bifida and neural tube defect in the developing foetus without side effects. Even a very small probability of disease justifies the treatment, and so the decision to supplement all pregnant women is accepted [1]. Some other good decisions are self-evident, since clinicians are faced everyday with the problem. Nobody has any doubt about starting aspirin in patient with myocardial infarction despite the known side effects of aspirin.

When the physician is not sure about the correct diagnosis, and the treatment could be harmful, as a rule of thumb, based on the Hippocratic Oath, a patient should be treated only if the physician is confident that the expected benefits exceed the expected risks. Also the patient will comply with the treatment, only when convinced that the benefit exceeds the risk.

The aim of this note is to explain the elements to consider in order to complex decisions, with a specific focus on decision model theory for increasing the awareness of decision making in everyday clinical practice.

\section{How do clinicians make decisions?}

A. Would you start hypoglicemic therapy in a 52-yearold with a fasting plasma glucose level of $247 \mathrm{mg} / \mathrm{dl}$ ?

B. Would you start anticoagulation in a 90-year-old patient with atrial fibrillation along with renal failure, anemia, hypertension, diabetes, and a history of falls from orthostatic hypotension?

C. How would you treat the 80-year-old man in the case above? In the simple case (A), clinical experience can 
direct the decision: treat the patient. In the more complex case (B), the decision requires a trade off between benefit (stroke prevention) and risk (hemorrhagic complications). Finally, in the third case (C), we have to choose between different diagnoses that mandate different treatments, with different costs/ benefit ratios. In this case, decision models may be helpful. Decision models are simplified representations of a complex reality that include and organize different possible health statuses (diagnostic hypotheses), the possible different decisions (diagnostic tests and therapy), the possible different consequences, with their probabilities and utilities.

The formal steps in medical decision making are the following:

1. formulation of diagnostic hypotheses and clinical strategies;

2. assignment of a probability to each hypothesis;

3. evaluation of the information of each diagnostic test;

4. evaluation of benefits and costs of each therapy;

5. computation of expected utility of each strategy.

These steps can be represented as a decision tree, a graphical tool of decision analysis.

\section{A probabilistic approach to diagnosis}

The probabilistic approach considers the diagnostic problem as a continuous update of the evidence from which we start. Clinicians formulate diagnostic hypotheses, and assign probability of being true to each one. Then they continuously revise the probabilities in view of new evidence coming from information given by diagnostic tests.

Coming back to the 80 -year-old patient, we need to assign a probability to each hypothesized diagnosis: (pneumonia, COPD acute exacerbation, heart failure, acute pulmonary embolism) considering that each disease must exclude any other, and the probabilities assigned must sum up to 1.0 (to $100 \%$ ). For our patient, we identified four possible diagnoses, but to be really exhaustive we cannot exclude a fifth category, that comprises something unexpected that could be reappraised in the subsequent diagnostic work-up, remembering that the main cause of diagnostic error is omission: "It never crossed my mind!".

That is our scenario:

1. Pneumonia

2. Pulmonary embolism

3. COPD acute exacerbation

4. Heart failure

5. Other
Giving a value of probability (at least a range of probabilities) to each diagnosis is a hard but crucial step. In this way, we set the pre-test probability of the patient as a starting point of the process: this is the probability that the clinician assigns to the "event" that the patient actually has that particular disease using his clinical judgement, before performing any diagnostic test.

Pre-test probability can be best estimated by the physician according to personal experience or considering the relative frequencies (prevalences) of the different hypothesized conditions in the population presenting with the signs, symptoms and history reported by that particular patient.

For example, Diehr et al. [2] report that in 483 patients admitted to the emergency department (ED) complaining of cough, $2.6 \%$ have pneumonia, documented by radiogram.

Pre-test probability can also be estimated using published and validated clinical prediction rules (CPR). They are algorithms that allow the estimate of probability of disease given some clinical characteristics. Many clinical scores exist to quantify the pulmonary embolism pre-test probability [3] and other suspected conditions [4, 5].

For example, considering pulmonary embolism and pneumonia predicting rules for our patient, the pre-test probabilities are about 16 and $40 \%$, respectively, according to Wells and Heckerling [6, 7].

\section{To treat or not to treat?}

When pre-test probability has been assigned, the clinician has to decide if the patient must be treated or not. Obviously, if the physician is highly confident (for example 99\%) that the patient has pulmonary embolism (e.g. $99 \%$ of pre-test probability), then he should treat the patient as soon as possible. Conversely, if he is quite certain that the patient is not diseased (e.g. $1 \%$ of pre-test probability) he would not treat him for pulmonary embolism. For intermediate values he can order diagnostic tests to gain more information about the real health status of the patient, depending on how much informative could be the programmed test.

How are decision thresholds defined? In other words, when is the probability of pulmonary embolism in our patient so low as to avoid therapy, or high enough to immediately start the treatment? Many aspects must be considered, such as the severity of disease (e.g. the risk of death or serious events for untreated patients), the efficacy of the therapy (benefits) and the side effects of the therapy (costs).

If the pre-test probability for a specific disease is above the decision threshold, and the benefits of the treatment 
Fig. 1 Therapy effectiveness and safety, utility and cost/ benefit ratio (data reported in the $2 \times 2$ table are derived from [8])
The treatment threshold, $\mathrm{p}^{*}$, can be defined as

$\mathrm{p}^{*}=(\mathrm{C} / \mathrm{B}) /(\mathrm{C} / \mathrm{B}+1)$

where $C$ are the costs and $B$ the benefits.

If the probability of disease of a patient exceeds $\mathrm{p}^{*}$ then the best decision will be "to treat", because for that patient the expected benefits of treatment are greater than the expected costs.

Costs and benefits can be estimated considering:

1. the risk of death for PE in untreated patients;

2. the risk of death for $P E$ in treated patients;

3. the risk of death for side effects of treatment.

so to estimate utilities of patients.

Given the literature efficacy data,the risk of death of an untreated patient with PE is $30 \%$. Treatment with unfractionated heparin or Low Molecular Weight heparin followed by oral anticoagulant reduces the risk of death to $8 \%$ [8]. Oral anticoagulant therapy brings a risk of haemorrhage with a mortality rate of $1 \%$.[11]

\section{Death}

$\begin{array}{rccc} & \text { Yes } & \text { No } & \text { Total } \\ \text { Heparin } & 8 & 92 & 100 \\ \text { Placebo } & 30 & 70 & 100 \\ \text { Total } & 38 & 162 & 200\end{array}$

Risk of death in heparinpatients: $8 / 100=0.08=8 \%$

Risk of death in placebogroup: $\quad 30 / 100=0.30=30 \%$

ARR (Absolute Risk reduction): $\quad 0.30-0.08=0.22=22 \%$

NNT (Number Needed to Treat): $\quad 1 / A R R=1 / 0.22=5$

RR (Relative Risk): $\quad 0.08 / 0.30=0.267$

OR (Odds Ratio): $\quad 8^{\star} 70 / 92^{*} 30=0.203$

Utility is a number between 1 (perfect health) and 0 (death).

- Utility of non-diseased non-treated $\left(U_{\mathrm{dt}}\right)$ is set to 1 (healthy person without treatment harm)

- Utility of diseased patients treated $\left(U_{D T}\right)$ must take into account both efficacy and side effects of treatment. Specifically, it considers the utility of perfect health $\left(\mathrm{U}_{\mathrm{dt}}=1\right)$ the risk of death for pulmonary embolism in treated patients $(0.08)$, the risk of death for haemorrhage in treated patients $(0.01)$, the probability of survival of treated patients (1-risk of death for of treated patients, 0.92), and the risk of death for haemorrhage in treated patients who survive to pulmonary embolism $\left(0.92^{*} 0.01\right)$ According to the above reported risk estimates, the utility of diseased treated can be calculated as $: \mathrm{U}_{\mathrm{DT}}=1-\left(0.08+0.92^{*} 0.01\right)=0.91$.

- Utility of non-diseasedpatients treated $\left(U_{\mathrm{aT}}\right)$ must take into account the side effects of oral anticoagulant therapy (risk of death for haemorrhage) $\mathrm{U}_{\mathrm{dT}}=1$ $0.01=0.99$

- Utility of diseased patients non-treated $\left(\mathrm{U}_{\mathrm{D} t}\right)$ is calculated starting from the risk of death for pulmonary embolism(0.30): $U_{D t}=1-0.3=0.7$

The costs $(\mathrm{C})$ of the treatment are, by definition, those faced by non diseased patients. They can be calculated as the difference between the utility of non-diseased non-treated $\left(\mathrm{U}_{\mathrm{dt}}\right)$ and the utility of non-diseased treated $\left(\mathrm{U}_{\mathrm{dT}}\right)$ patients: $\mathrm{C}=\mathrm{U}_{\mathrm{dt}}-\mathrm{U}_{\mathrm{dT}}=1-0.99=0.01$. In this case they correspond to the risk of death for haemorrhage in treated patients.

The benefits $(B)$ are defined as the net benefit that the treatment brings to diseased patients. They can be calculated as the difference between the utility of diseased treated $\left(U_{D T}\right)$ and the utility of diseased non-treated $\left(U_{D t}\right)$ patients: $B=U_{D T}-U_{D t}=0.91-0.7=0.21$.

According to the data of our example, the treatment threshold, $\mathrm{p}^{*}$, is then: $\mathrm{p}^{*}=(\mathrm{C} / \mathrm{B}) /(1+\mathrm{C} / \mathrm{B})=(0.01 / 0.21) /((0.01 / 0.21)+1)=0.0454=4.5 \%$ 
Fig. 2 Diagnostic accuracy and decision thresholds (data in the $2 \times 2$ table are adapted from [14])

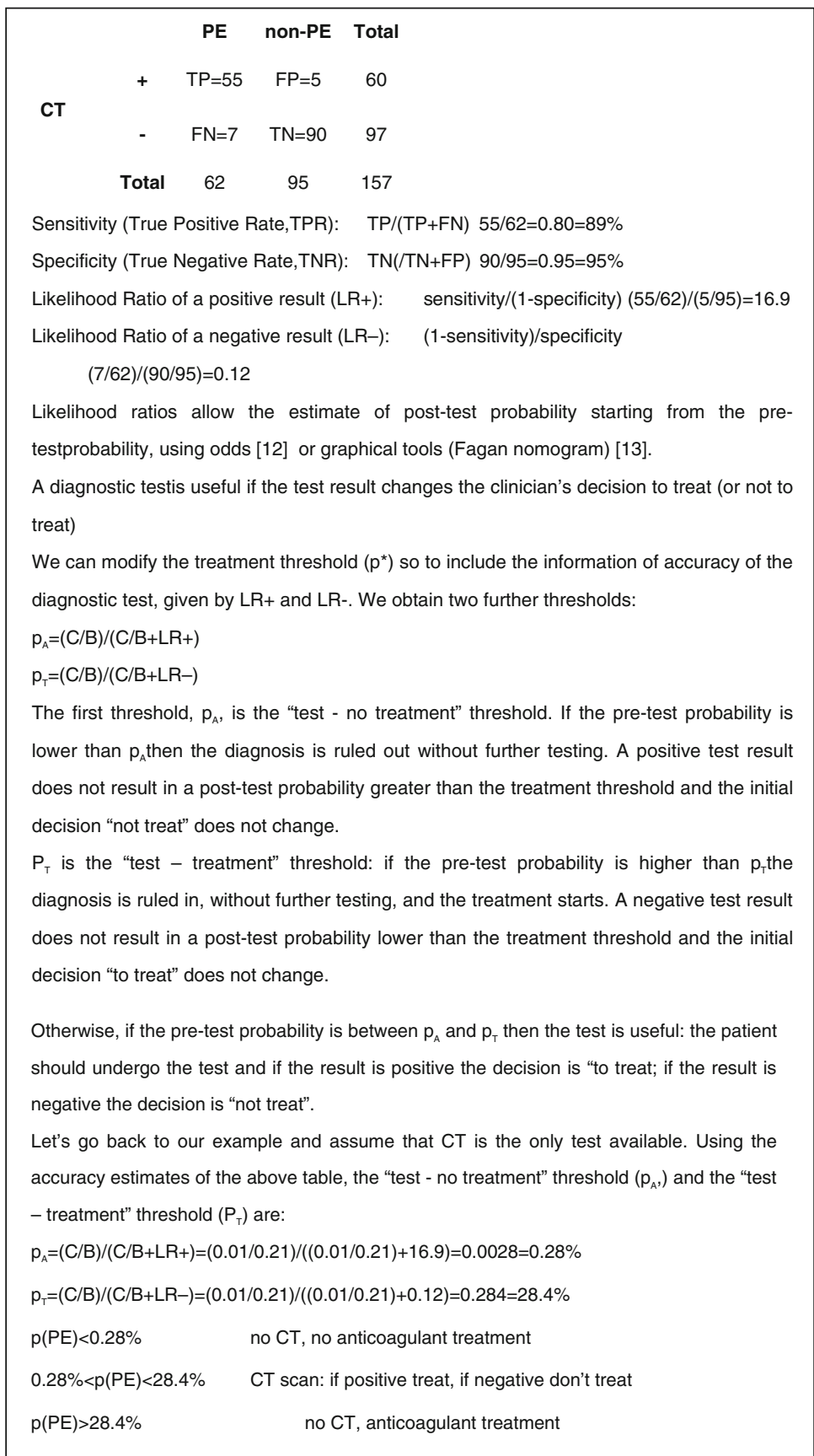

outweigh the harm, the decision will be to treat. On the contrary, if the patient pre-test probability is below the decision threshold, the decision will be not to treat.

Figure 1 reports the estimation of decision threshold for starting heparin treatment in a patient with pulmonary embolism. The death rate in untreated patients affected by pulmonary embolism is about $30 \%$, while treatment lowers mortality to about $8 \%$. The risk of death from haemorrhagic events is about $1 \%$; we can derive a decision threshold of $5 \%$. If the pre-test probability of pulmonary embolism in our patients is above $5 \%$, to treat the patient would cause more benefit than harm [8].

\section{To test or not to test?}

In order to maximise benefits (ideally try to treat only and all the diseased patients) and minimise harm (ideally do not treat any undiseased patients) we can order diagnostic tests (ideally a perfect one: $100 \%$ sensitive and $100 \%$ specific). 
In real life, a perfect test does not exist, and to be really useful, a diagnostic test must be used only if its result can change the prior decision based on the pre-test probability. Starting from a pre-test probability greater than the decision threshold, a negative test result should lower the probability of disease (post-test) below the decision threshold, leading to a "not to treat" decision. Conversely, in case of a pre-test probability below the decision threshold, a positive test result should lead the patient posttest probability to cross the decision threshold, resulting in a "to treat" decision.

Consider a pre-test probability of heart failure of the patient above, to be about $10 \%$ (based on clinical gestalt). If we order a blood sample for Brain Natriuretic Peptide (BNP) with a sensitivity of $97 \%$ and a specificity of $62 \%$, and the test result is negative $(<50 \mathrm{pg} / \mathrm{ml})$, the post-probability would be less than $1 \%$. This value would lead to a "not to treat" decision [9].

For a formal definition of sensitivity and specificity of a test, and for considering how sensitivity and specificity can be used to compute the post-test probability and to define other useful decision thresholds see the Fig. 2.

\section{Decision tree and expected utilities}

To return to our case: To simplify, consider only the diagnosis of pulmonary embolism. As already seen in paragraph three, the pre-test probability of pulmonary embolism is $16 \%$. Choose the computed tomography (CT) as diagnostic test. Sensitivity and specificity are 89 and $96 \%$, respectively [10].

Using utility values of Fig. 1, we can try to build the decision tree, a graphical tool of decision analysis. Key elements of a decision tree are nodes and branches. Decision nodes (usually represented by squares) represent points in the tree where a decision must be made: the clinician must choose among two or more possible actions (treat, do not treat, test). Chance nodes (represented by circles) are points on the tree where probabilities must be considered: each possible outcome, indicated in a node, has a specified probability of occurrence. Terminal nodes are final points of every pathway: they represent the final outcome of each pathway. Branches are lines starting from a node (and usually connecting nodes). They indicate the outcome or the action chosen.

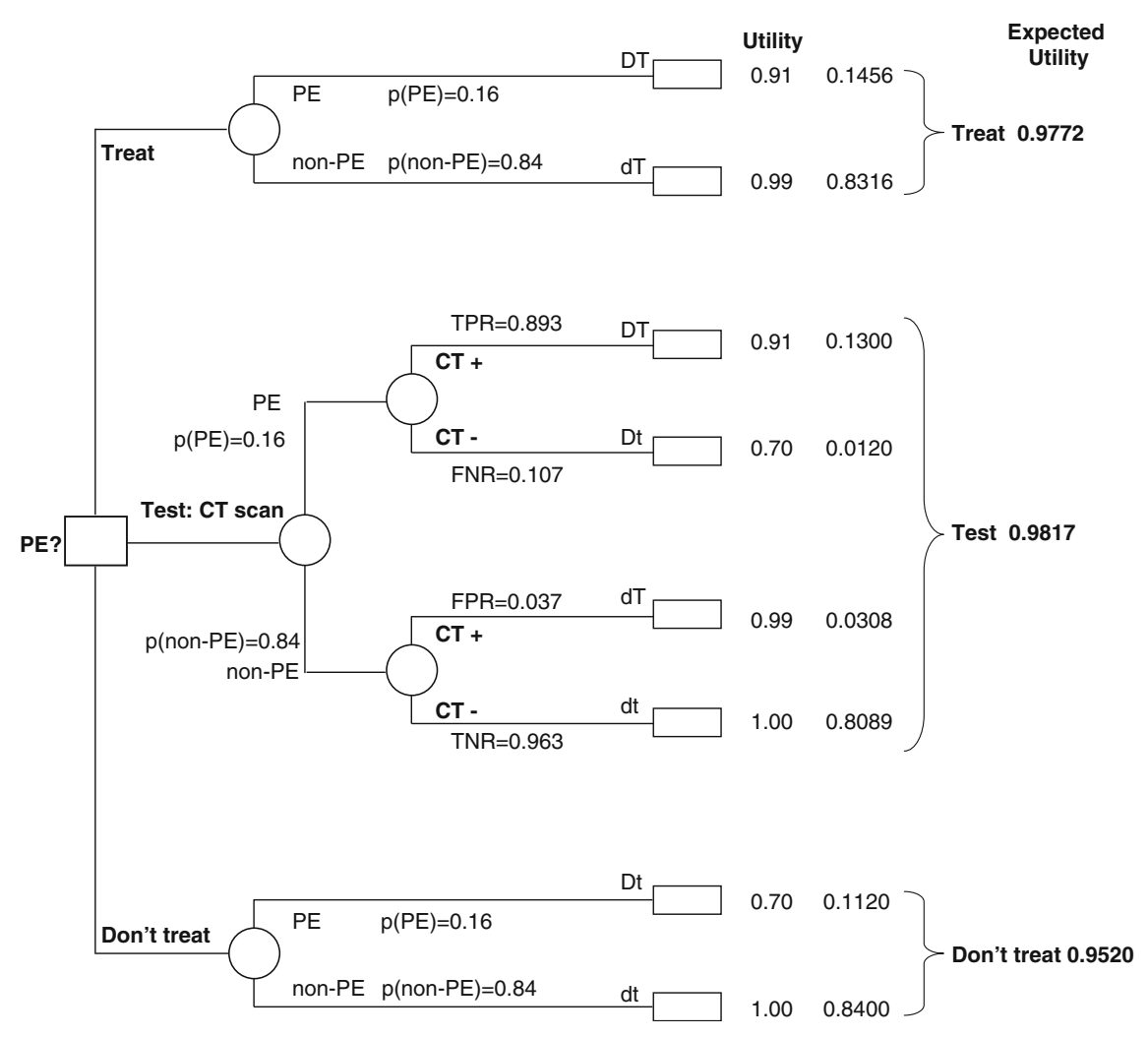

Fig. 3 Expected utilities are calculated by multiplying the utility of each branch by the probability of the branch and summing all the products obtained from the branches of the same strategy. For example, starting from a pre-test probability of $16 \%$, in the "Treat" strategy we would calculate the expected utility as: $0.16 \times 0.91+0.84 \times 0.99=0.9772 . P E$ pulmonary embolism, $C T$ computed tomography, $D$ diseased, $d$ not diseased, $T$ treated, $t$ not treated 
In conclusion, assuming an intermediate pre-test probability of PE (16\%) and CT as the only diagnostic test, we have proposed three strategies: treat all the patients; do not treat any patient; test and treat only positive patients. The best strategy, according to expected utility, would be the latter (Fig. 3).

\section{Take home message}

1. Think of possible diagnoses.

2. Assign pre-test probability.

3. Think of possible therapies, considering benefit and harm.

4. Think of possible diagnostic tests, considering Likelihood Ratios.

5. Compute the expected utilities (check if the guidelines you use are based on a correct decision making approach and critically appraise the evidence they use in deciding).

6. Build the decision tree summarising all of the above points.

\section{Conflict of interest None}

\section{References}

1. Centers for Disease Control and Prevention (CDC) (2010) CDC Grand Rounds: additional opportunities to prevent neural tube defects with folic acid fortification. MMWR Morb Mortal Wkly Rep 59(31):980-984

2. Diehr P, Wood RW, Bushyhead J, Krueger L, Wolcott B, Tompkins RK (1984) Prediction of pneumonia in outpatients with acute cough—a statistical approach. J Chronic Dis 37(3):215-225
3. Ceriani E, Combescure C, Le Gal G, Nendaz M, Perneger T, Bounameaux H, Perrier A, Righini M (2010) Clinical prediction rules for pulmonary embolism: a systematic review and metaanalysis. J Thromb Haemost 8(5):957-970

4. Lenzi T, Tampieri A, Cantarini MC (2010) Scores clinici in medicina d'urgenza. Edizioni Medico-Scientifiche, Torino

5. Ebell MH (2001) Evidence-based diagnosis: a handbook of clinical prediction rules. Springer, New York

6. Wells PS, Anderson DR, Rodger M, Ginsberg JS, Kearon C, Gent M, Turpie AG, Bormanis J, Weitz J, Chamberlain M, Bowie D, Barnes D, Hirsh J (2000) Derivation of a simple clinical model to categorize patients probability of pulmonary embolism: increasing the models utility with the SimpliRED D-dimer. J Thromb Haemost 83(3):416-420

7. Heckerling PS, Tape TG, Wigton RS, Hissong KK, Leikin JB, Ornato JP, Cameron JL, Racht EM (1990) Clinical prediction rule for pulmonary infiltrates. Ann Intern Med 113(9):664-670

8. European Society of Cardiology Task Force on Pulmonary Embolism (2008) Guidelines on diagnosis and management of acute pulmonary embolism. Eur Heart J 29:2276-2315

9. Maisel A, Krishnaswamy P, Nowak R, McCord J, Hollander J, Duc P, Omland T, Storrow A, Abraham W, Wu A, Clopton P, Steg P, Westheim A, Knudsen C, Perez A, Kazanegra R, Herrmann H, McCullough P (2002) Rapid measurement of B-type natriuretic peptide in the emergency diagnosis of heart failure. N Engl J Med 347(3):161-167

10. Roy PM, Colombet I, Durieux P, Chatellier G, Sors H, Meyer G (2005) Systematic review and meta-analysis of strategies for the diagnosis of suspected pulmonary embolism. BMJ 331(7511):259

11. Linkins LA, Choi PT, Douketis JD (2003) Clinical impact of bleeding in patients taking oral anticoagulant therapy for venous thromboembolism: a meta-analysis. Ann Intern Med 139(11): 893-900

12. Deeks JJ, Altman DG (2004) Diagnostic tests 4: likelihood ratios. BMJ 329(7458):168-169

13. Fagan TJ (1975) Nomogram for Bayes theorem. N Engl J Med 293(5):257

14. Qanadli SD, Hajjam ME, Mesurolle B, Barré O, Bruckert F, Joseph T, Mignon F, Vieillard-Baron A, Dubourg O, Lacombe P (2000) Pulmonary embolism detection: prospective evaluation of dual-section helical CT versus selective pulmonary arteriography in 157 patients. Radiology 217(2):447-455 